Review

\title{
Dynamic materials fabricated from water soluble pillar[n]arenes bearing triethylene oxide groups
}

\author{
Tangxin Xiao ${ }^{\mathrm{a}, *}$, Ling Zhou ${ }^{\mathrm{a}}$, Lixiang $\mathrm{Xu}^{\mathrm{a}}$, Weiwei Zhong, Wei Zhao ${ }^{\mathrm{a}}$, Xiao-Qiang Sun ${ }^{\mathrm{a}}$, \\ Robert B.P. Elmes ${ }^{\text {b,* }}$
}

a School of Petrochemical Engineering, Advanced Catalysis and Green Manufacturing Collaborative Innovation Center, Changzhou University,

Changzhou 213164, China

b Department of Chemistry, Maynooth University, National University of Ireland, Maynooth, Ireland

\section{A R T I C L E IN F O}

\section{Article history:}

Received 29 April 2018

Received in revised form 17 May 2018

Accepted 24 May 2018

Available online 24 May 2018

\section{Keywords:}

Pillar[n]arenes

Host-guest interactions

LCST

Thermoresponsive materials

Smart window

\section{A B S T R A C T}

Pillar[n]arenes are a new kind of supramolecular macrocyclic hosts which have developed rapidly due to their unique topology and high functionality, giving rise to many applications in the construction of interesting and functional materials. Among them, water-soluble pillar[n]arenes bearing triethylene oxide (TEO) chains have drawn increasing research interest due to their advantageous properties. In this review, we summarized the recent progress of dynamic materials fabricated from water soluble pillar[n] arenes bearing TEO groups, including thermoresponsive materials with lower critical solution temperature (LCST) behavior, cyclic host liquids, and smart windows. It is anticipated that more and more 'smart' supramolecular materials based on modified pillar[n]arenes will be developed in this burgeoning area of research.

(C) 2018 Chinese Chemical Society and Institute of Materia Medica, Chinese Academy of Medical Sciences. Published by Elsevier B.V. All rights reserved.

\section{Introduction}

Host-guest interactions based on a macrocyclic host and a guest molecule have played an important role in supramolecular chemistry due to their various dynamic properties [1-6]. Incorporation of host-guest interactions can endow materials with interesting properties, such as stimuli responsiveness, selfhealing, and adaptability. Macrocyclic host molecules, such as crown ethers, calixarenes, cyclodextrins and cucurbiturils, are typical host molecules which are widely used to achieve so-called 'smart' materials [7,8]. Pillar[n]arenes, as a new kind of macrocyclic host in supramolecular chemistry, have developed rapidly and attracted much attention in creating various supramolecular assemblies with application as supramolecular materials [9-18]. In contrast to typical host molecules, pillar[n]arenes display many useful features. Where this highly symmetrical pillar shaped architecture display similarities to the pumpkin-shaped cucurbiturils $[19,20]$, the repeating units of pillar[n]arenes are also similar to calixarenes [21-23]. Furthermore, pillar[n]arenes have numerous phenolic oxygen units on both upper and lower rims, making

\footnotetext{
* Corresponding authors.

E-mail addresses: xiaotangxin@cczu.edu.cn (T. Xiao), robert.elmes@mu.ie (R.B.P. Elmes)
}

them analogous to the highly functionalized cyclodextrins [24-26]. Pillar[n]arenes are easy to prepare, facile to modify, possess a unique shape and display solubility in a range of solvents - many of the properties required from a desirable macrocyclic host. In particular, their symmetrical pillar structure and convenient modification endows them with the ability to bind a diverse range of guest molecules - neutral guests, anionic guests, cationic guests, rigid guests, flexible chain guests, hydrophobic guests and hydrophilic guests can all be accommodated with pillar[n]arenes $[11,27]$.

Stimuli-responsive materials that can respond to external stimuli, such as temperature, light, redox reactions and $\mathrm{pH}$, have also received much attention in the field of smart materials due to their various practical applications. Among these materials, watersoluble thermoresponsive materials exhibiting lower critical solution temperature (LCST) behavior are some of the most fascinating materials due to their important potential biomedical applications. At temperatures higher than the LCST, these materials display decreased solubility, leading to a fall in solution transmittance and an observable cloud point. Generally, most investigations on LCST phase behavior are focused on polymers, such as poly ( $N$-isopropylacrylamide), however, in recent years LCST behavior has gradually been reported for host-guest systems, focusing mainly on pillar[n]arene systems. Compared with polymers, these pillar[n]arene based materials not only possess LCST behavior but 
also show additional functions, such as controllable host-guest behavior. Interestingly, pillar[n]arene derivatives bearing triethylene oxide (TEO) chains exhibit LCST behavior in aqueous solution, which seems to be a common feature of LCST behavior of ethylene oxide dendronized polymers [28]. In contrast, pillar[n]arenes with short ethylene oxide chains have been found to be insoluble in water. Moreover, LCST behavior is not observed for model compounds without the pillar[n]arene backbone, suggesting that the pillar-shaped hydrophobic macrocyclic architecture containing a number of hydrophilic TEO groups is required for LCST behavior [29]. It is also worth noting that the ionic nature of some watersoluble pillar[n]arenes can affect electrolyte behavior [30,31], but those pillar[n]arenes bearing TEO chains are non-ionic and retain their water-solubility overcoming any such limitations to responsive LCST behavior. In addition, pillar[n]arenes bearing TEO chains exhibit many other interesting properties. For example, they can serve as cyclic host liquids for highly efficient construction of mechanically interlocked molecules [32]. In this mini-review, we will describe the recent advances in dynamic materials constructed from pillar[n]arenes bearing TEO chains.

\section{Materials with LCST behavior}

As mentioned above, pillar[n]arenes exhibit lower LCST behavior due to modification of multiple TEO groups. In 2012, Ogoshi et al. reported the first example of pillar[5]arene bearing ten TEO chains that exhibited LCST behavior [29]. As we can see in Fig. 1, the clear aqueous solution of P5 at room temperature became turbid on heating before becoming transparent again upon cooling. At temperatures above the cloud point ( $\left.T_{\text {cloud }}\right)$, the interaction of the hydrophobic groups causes P5 to aggregate and then separate from water. The $T_{\text {cloud }}$ was determined to be $42^{\circ} \mathrm{C}$ in $2 \mathrm{mmol} / \mathrm{L}$ aqueous solution of P5. Notably, $T_{\text {cloud }}$ was dependent upon the concentration of P5. As the concentration of P5 increased, $T_{\text {cloud }}$ decreased. Interestingly, the $T_{\text {cloud }}$ of $\mathbf{P 5}$ can be readily tuned from $42^{\circ} \mathrm{C}$ to $60^{\circ} \mathrm{C}$ upon addition of didecylviologen dibromide (C10Vio) as a guest. When C10Vio was added, the turbid solution of P5 became clear at $55^{\circ} \mathrm{C}$ (Fig. 1b). Furthermore, if cucurbit[7]uril was used as a competitive host to exclude C10Vio from the cavity of P5, $T_{\text {cloud }}$ was recovered to that of pristine P5, followed by the observation of a turbid solution. This chemically responsive material may have potential use for cloud point extraction.

Controlling supramolecular assemblies on the nanoscale by external stimuli has drawn much attention in nanoscience. Among various stimuli, light is especially useful due to its rapid and noninvasive nature. Azobenzene derivatives have proven to be especially advantageous due to their controllable conformational changes between the trans and cis forms with UV/visible light irradiation, accompanied by variations in their molecular properties. In a follow-up investigation, Ogoshi et al. demonstrated the photoreversible switching of LCST behavior in a photoresponsive host-guest system, constructed from pillar[6]arene with TEO substituents and an azobenzene derivative [33]. They first synthesized a new water-soluble thermoresponsive pillar[6]arene with 12 TEO groups (P6, Fig. 2a). Similar to P5, P6 also exhibits LCST behavior in aqueous solution. Host-guest studies showed that the trans form of an azobenzene guest (G1, Fig. 2a) formed a stable 1:1 complex with P6. In a $2 \mathrm{mmol} / \mathrm{L}$ aqueous solution of P6, complexation increased the $T_{\text {cloud }}$ from $41^{\circ} \mathrm{C}$ to $57^{\circ} \mathrm{C}$. Irradiation with UV light induced a conformational change for $\mathbf{G 1}$ from the trans to cis form, and dethreading occurred due to a size mismatch between the cis form of $\mathbf{G 1}$ and the pillar[6]arene cavity. Subsequently, this dethreading decreased the $T_{\text {cloud }}$ from $57^{\circ} \mathrm{C}$ to $41^{\circ} \mathrm{C}$. This process was reversible, and the cloud point could be switched by alternating irradiation with UV/visible light. In this work, the photocontrollable threading/dethreading resulted in photoreversible switching of LCST behavior, exhibiting their potential application for drug delivery.

In 2014, Xue et al. reported a new water-soluble thermoresponsive pillar[10]arene with TEO groups, the cloud point of which could be reversibly controlled based on a chemical-responsive host-guest system (Fig. 3) [34]. By etherification of per-hydroxylated pillar[10]arene, P10 was obtained as a light yellow liquid. Like pillar[5,6]arenes with TEO groups described above, P10 also showed LCST behavior in water. The clear aqueous solution of P10 $(2.00 \mathrm{mmol} / \mathrm{L})$ became turbid upon heating to $50^{\circ} \mathrm{C}$. It became clear again as the temperature decreased. Formation of a stable host-guest complex based on P10 and G2 was evidenced by ${ }^{1} \mathrm{H}$ NMR spectroscopy, UV-vis absorption spectroscopy and isothermal titration calorimetry. The authors demonstrated that the $T_{\text {cloud }}$ of P10 increased upon the gradual addition of G2, resulting in a

(a)

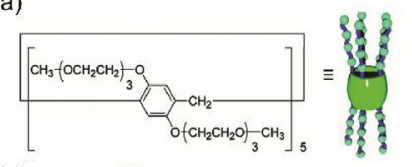

(b)

P5
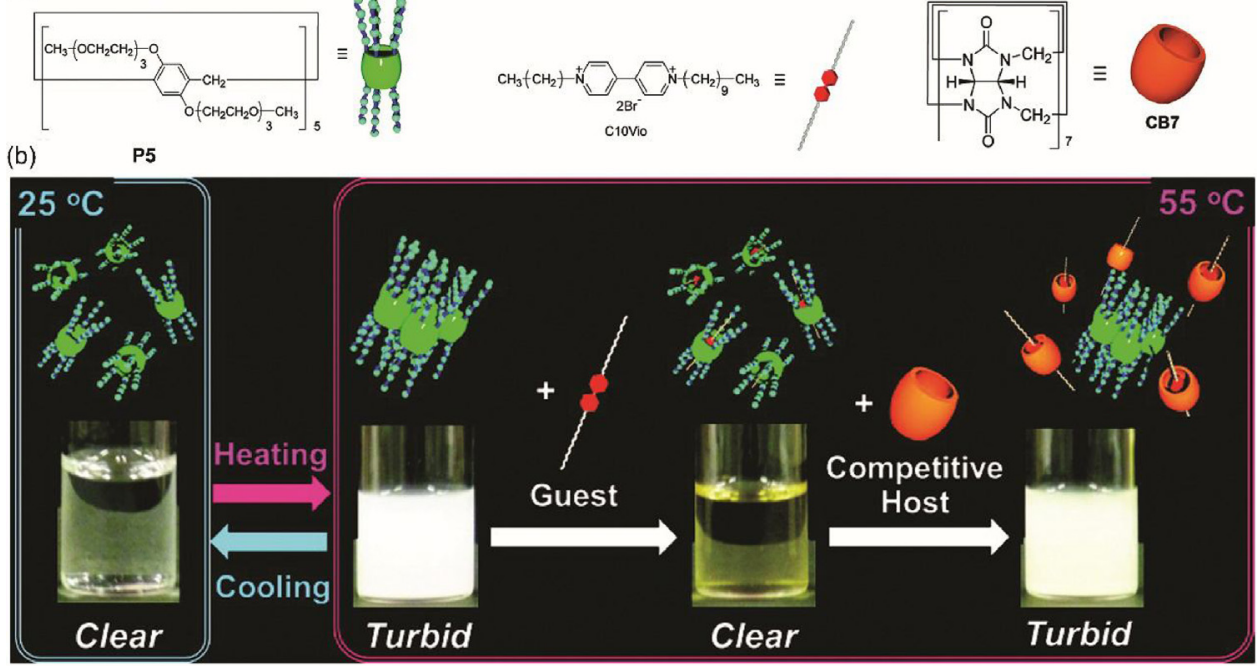

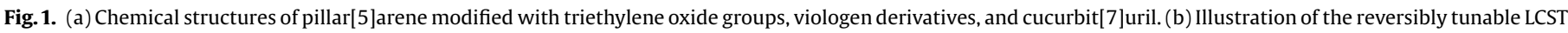
utilizing host-guest complexation of P5. Reproduced with permission [29]. Copyright 2012, American Chemical Society. 
(a)

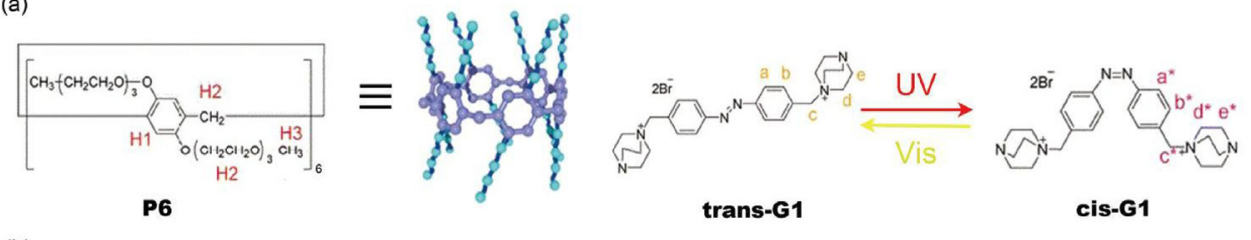

(b)

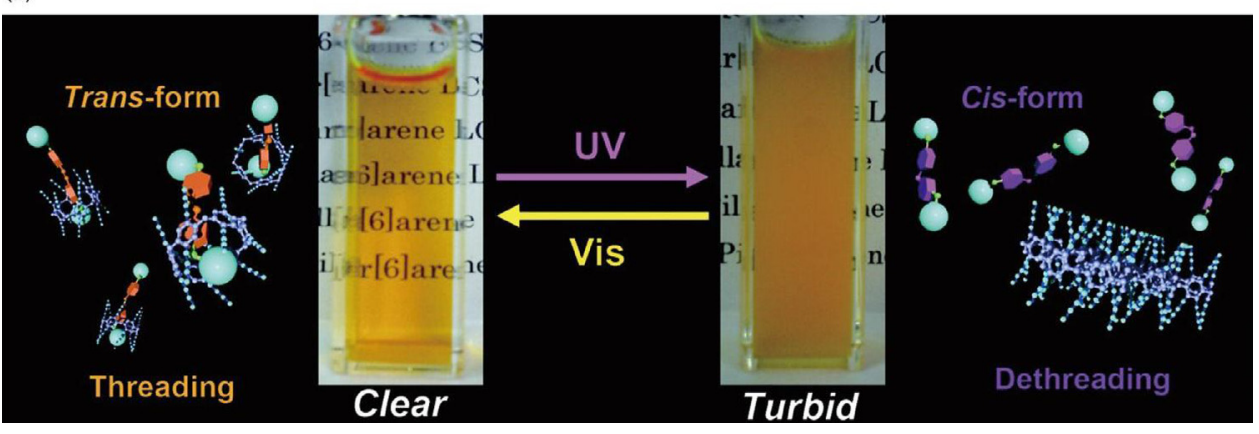

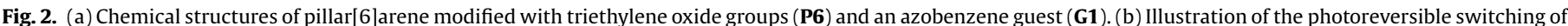
LCST utilizing host-guest complexation of P6. Reproduced with permission [33]. Copyright 2012, American Chemical Society.

clear solution (Fig. 3b). When excess diethylamine (DEA) was added to the above solution, the yellow solution turned dark green, indicating dissociation of the complex. Then the complex formed again when trifluoroacetic acid (TFA) was added to neutralize DEA. Concomitantly, the dark green solution gradually reverted to yellow. This process could be repeated several times (Fig. 3c). The

(a)

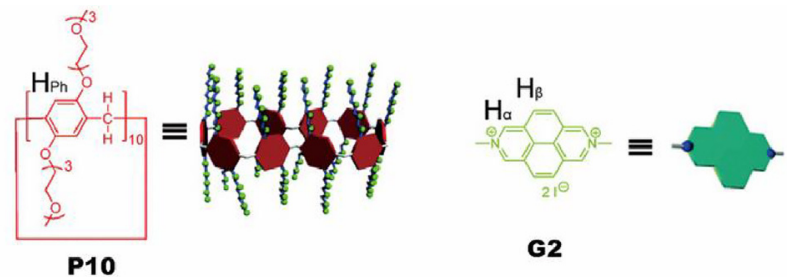

(b)

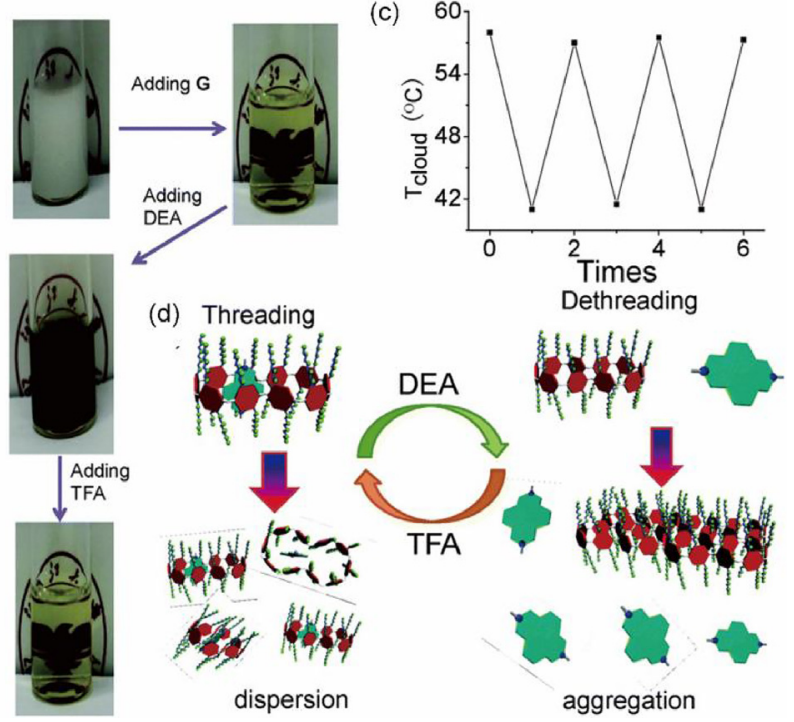

Fig. 3. (a) Chemical structures of P10 and G2. (b) Photos of a mixture of P10 (2.00 $\mathrm{mmol} / \mathrm{L})$ and $\mathbf{G 2}(3.00 \mathrm{mmol} / \mathrm{L})$ in water at $50^{\circ} \mathrm{C}$ after adding DEA or TFA. (c) Changes in $T_{\text {cloud }}$ upon addition of DEA or TFA. (d) Illustration of chemicalresponsive control of the LCST behavior by pillar[10]arene-based host-guest interactions. Reproduced with permission [34]. Copyright 2014, the Royal Society of Chemistry. authors suggest that this thermoresponsive material has potential use in many bio-relevant fields, such as smart bioactive surfaces, phase separation immuno-assays, hyperthermia induced drug delivery and tissue engineering due to the biocompatible nature of the TEO moieties.

Macromolecular supra-amphiphiles refer to a kind of macromolecular amphiphile whose hydrophobic and hydrophilic parts are linked by supramolecular forces. In 2015, Huang et al. reported a new host-guest recognition motif between a thermoresponsive water-soluble pillar[7]arene (P7) and an azobenzene derivative (Fig. 4) [35]. P7 was functionalized with 14 TEO groups and also showed LCST behavior in water. The authors used this recognition motif to fabricate a pillararene-based supra-amphiphilic polypseudorotaxane, which can further self-assemble into vesicles in water. This new host-guest system in water has dual photo- and thermo-responsiveness. The host-guest complexation between P7 and the model compound 1 was first investigated by ${ }^{1} \mathrm{H}$ NMR spectroscopy and ITC. The stoichiometry and binding constant between $\mathbf{P 7}$ and trans-1 was determined to be $1: 1$ and $(1.97 \pm 0.24)$ $\times 10^{4} \mathrm{~L} / \mathrm{mol}$. The photocontrollable threading/dethreading between $\mathbf{P 7}$ and $\mathbf{1}$ could be controlled by alternative irradiation with light at $435 \mathrm{~nm}$ and $365 \mathrm{~nm}$. Moreover, based on the LCST behavior of P7, the disassembly and assembly process of the complex P7 $\supset$ trans-1 could also be switched by the heating/cooling treatment. Subsequently, the self-assembly of random copolymer 2 and P7 was studied by a combination of techniques, such as NOESY, DLS and TEM. As a result, the reversible transformations between nanoparticles based on the self-assembly of $\mathbf{2}$ and vesicles based on the self-assembly of P7 $\supset \mathbf{2}$ were achieved by controlling the temperature or UV/vis light irradiation. Furthermore, the vesicles were successfully employed in the controlled release of calcein dye molecules. This example provided a new approach to combine polymer science with pillararene chemistry to fabricate functional supramolecular materials.

In a follow-up investigation, Huang et al. developed a dualthermoresponsive gemini-type supra-amphiphilic macromolecular [3]pseudorotaxane based on pillar[10]arene /paraquat cooperative complexation [36]. They chose pillar[10]arene with TEO groups (P10) as the host, which can bind two paraquat moieties to form a gemini-type supra-amphiphilic [3]pseudorotaxane $\left(\mathbf{P 1 0} \supset \mathbf{G}_{2}\right)$ (Fig. 5). The guest molecule G3 is paraquat-containing 


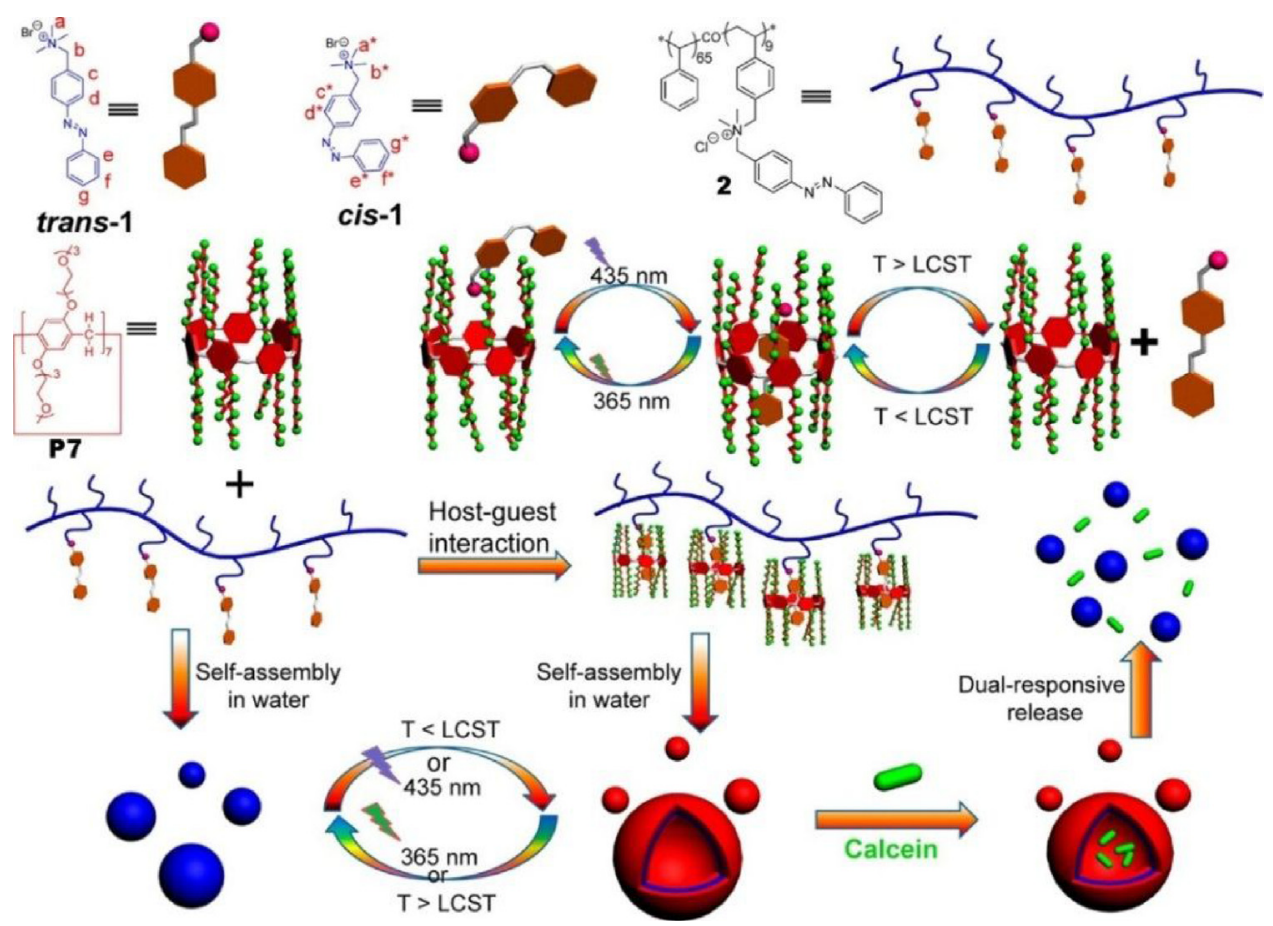

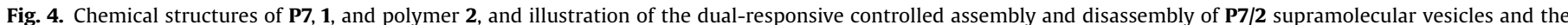
process of dual-responsive release of calcein molecules. Reproduced with permission [35]. Copyright 2015, American Chemical Society.

poly( $N$-isopropylacrylamide) (PNIPAM) polymers. Both P10 and G3 exhibits LCST behavior in water. Generally, $T_{\text {cloud }}$ of the host was affected by the addition of guest molecules. The effect of the added P10 on the thermoresponsive behavior of $\mathbf{G} 3$ was explored by

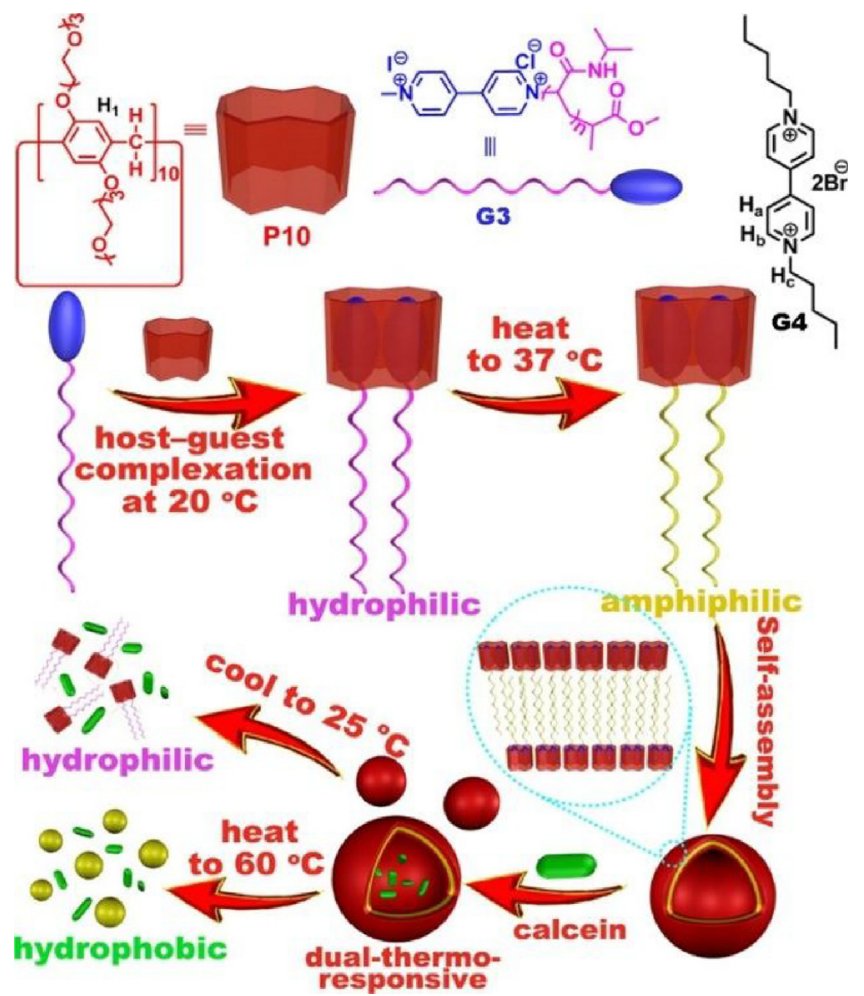

Fig. 5. Chemical structures of P10, G4 and polymer G3 and schematic illustration of preparation of polymeric vesicles and their application in dual-thermoresponsive release of calcein molecules Reproduced with permission [36]. Copyright 2016, American Chemical Society. turbidity experiments. A solution $\mathbf{P} \mathbf{1 0} \supset \mathbf{G}_{2}$ was prepared in water at $20^{\circ} \mathrm{C}$, and then gradually warmed to $37^{\circ} \mathrm{C}$ (above the LCST of PNIPAM), leading to the formation of vesicles evidenced by TEM and DLS. This is because the PNIPAM was changed from hydrophilic to hydrophobic upon increasing the temperature. Concomitantly, the water-soluble [3]pseudorotaxane was turned into an amphiphilic gemini-type supra-amphiphile. When a solution of vesicles was cooled to $25^{\circ} \mathrm{C}$, the hydrophobic part of $\mathbf{P 1 0} \supset \mathbf{G}_{2}$ became hydrophilic, and the average diameter of the assemblies decreased from $142 \mathrm{~nm}$ to $6.8 \mathrm{~nm}$, indicating the fragmentation of vesicles at $25^{\circ} \mathrm{C}$. By contrast, when the temperature was increased to $60^{\circ} \mathrm{C}$, the average size of the aggregates increased from $142 \mathrm{~nm}$ to $1484 \mathrm{~nm}$. This was due to the fact that phase separation had occurred when the temperature exceeded the LCST of P10, leading to irregular precipitates being observed by TEM. Finally, the vesicles were further applied in the controlled release of hydrophilic dye calcein and hydrophobic anticancer drug DOX.

Macrocyclic receptors are a major cornerstone in supramolecular chemistry. Recently, Jiang et al. reported several naphtholbased molecular receptors, of which perbutyloxatub[4]arene (TA4) was particularly interesting [37-40]. Although TA4 does not belong to the pillar[ $n]$ arene family, it is of value to discuss TA4 in the context of LCST behavior for this review. In recent work, the same group reported the synthesis and host-guest properties of a

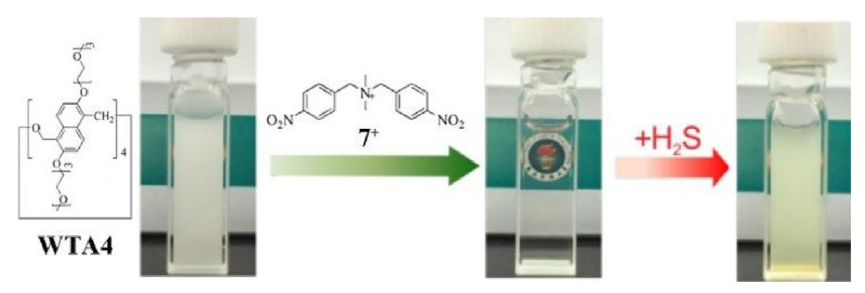

Fig. 6. Photos of WTA4 $(1.0 \mathrm{mmol} / \mathrm{L})$ in water before and after addition of $\mathbf{7}^{+}(2.0$ $\mathrm{mmol} / \mathrm{L})$ followed by $\mathrm{NaHS}(30.0 \mathrm{mmol} / \mathrm{L})$ in sequence at $43^{\circ} \mathrm{C}$. Reproduced with permission [41]. Copyright 2017, American Chemical Society. 
(a) Using Liquid Molecules

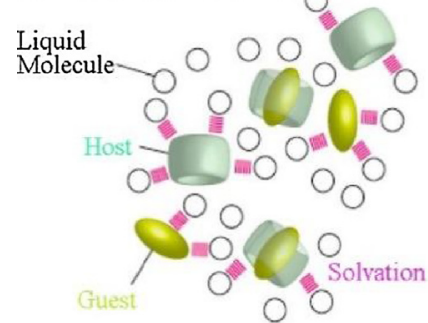

(c)

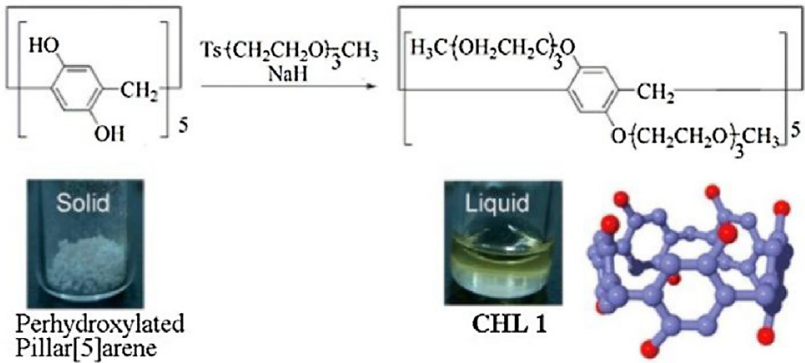

Fig. 7. Concept and synthesis of CHLs. (a) Host-guest complexation using liquid molecules as solvents. (b) Host-guest complexation in CHLs. (c) Synthesis and physical appearance of pillar[5]arene-based CHL1. Reproduced with permission [32]. Copyright 2012, American Chemical Society.

water-soluble oxatub[4]arene (WTA4) (Fig. 6) that showed $\mathrm{H}_{2} \mathrm{~S}$ responsive LCST behavior [41]. WTA4 exhibits high solubility in aqueous solution at $25^{\circ} \mathrm{C}$ and displayed reversible LCST behavior. The $T_{\text {cloud }}$ of WTA4 $(1 \mathrm{mmol} / \mathrm{L})$ was determined to be $35.3^{\circ} \mathrm{C}$. By careful screening, it was determined that guest $\mathbf{7}^{+}$could bind to WTA4 in water via a 1:1 binding stoichiometry. The effect of hostguest complexation on the $T_{\text {cloud }}$ of these systems was also studied. As the concentration of $\mathbf{7}^{+}$increased from 0 to $3 \mathrm{mmol} / \mathrm{L}$, the $T_{\text {cloud }}$ of WTA4 gradually increased from $35.3^{\circ} \mathrm{C}$ to $55.0^{\circ} \mathrm{C}$. On the basis of this host-guest system, chemically responsive turbid-to-clear and clear-to-turbid transitions can be achieved (Fig. 6). When a solution of WTA4 was heated to $43^{\circ} \mathrm{C}$ (above the $T_{\text {cloud }}$ of WTA4 $\left(35.3^{\circ} \mathrm{C}\right)$ ), the solution became milky. Addition of $\mathbf{7}^{+}$to the above solution at $43^{\circ} \mathrm{C}$ (below the $T_{\text {cloud }}$ of WTA4 $\supset \mathbf{7}^{+}\left(55^{\circ} \mathrm{C}\right)$ ) caused the solution to become transparent. The solution reverted to turbidity upon the addition of NaHS at $43^{\circ} \mathrm{C}$. This is thought to be due to the production of $\mathrm{H}_{2} \mathrm{~S}$ from NaHS and subsequent reduction of the nitro group of $\mathbf{7}^{+}$, leading to the destruction of the host-guest association between $\mathbf{7}^{+}$and WTA4. This work is particularly important as the LCST behavior of this new host-guest system responds to the occurrence of the important physiological gasotransmitter $\mathrm{H}_{2} \mathrm{~S}$.

\section{Cyclic host liquids}

Liquid media are commonly utilized for organic synthetic chemistry. By contrast to solid compounds, molecules in the liquid phase have more freedom and can react more easily with other molecules. To this end, Ogoshi et al. developed cyclic host liquids (CHLs) by transforming host molecules from solids to liquids at room temperature by modification with TEO groups [32]. This unique pillar[5]arene could not only play the role of host but also solvent when reacting with guest molecules and led to a highly efficient synthesis of mechanically interlocked molecules. As shown in Fig. 7, guest molecules could be directly surrounded by an excess of CHLs. While perhydroxylated pillar[5]arene is a solid, the pillar[5]arene modified with TEO groups (CHL1) is a liquid at room temperature. Introduction of flexible TEO chains reduces the crystallinity of pillar[5]arene and leads to a liquid state. CHL1 has good solubility both in water and numerous organic solvents, indicating that CHL1 could be employed as a solvent for a diverse range of compounds. DSC measurements showed that CHL1 remained as a liquid even at $-50^{\circ} \mathrm{C}$. In contrast to a typical

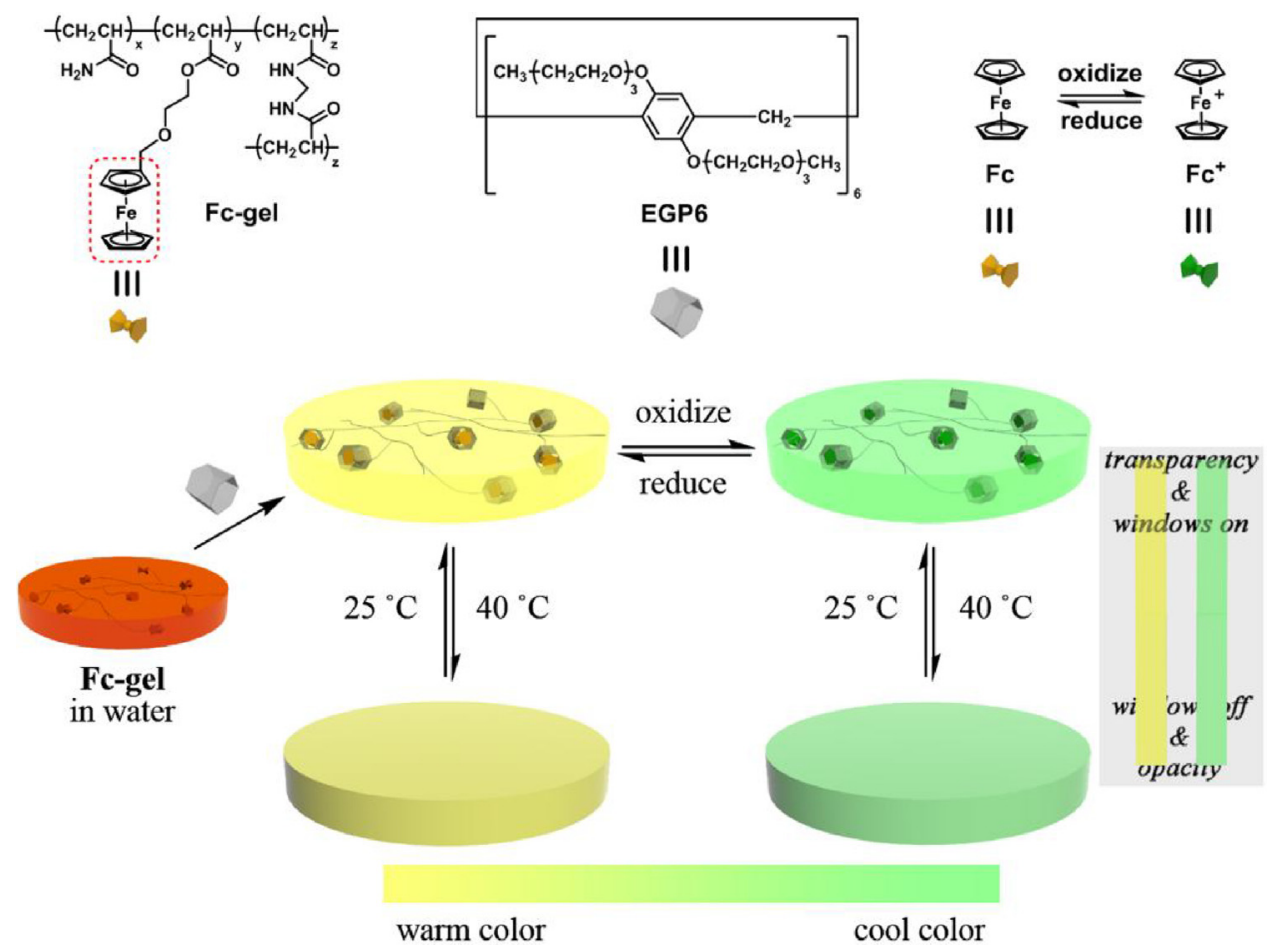

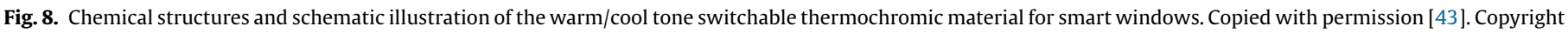
2018, Nature Publishing Group. 
complexation system, the CHL system is extremely efficient for maintaining host-guest complexation due to no addition of solvent being required. In this work, they demonstrated facile synthesis of [2]rotaxanes in a high yield by using CHL1 even with unfavorable statistical combinations of host-guest complexes.

By combining the advantages of polymer science and mechanically interlocked molecules, polyrotaxanes are fascinating supramolecular architectures, in which many macrocyclic molecules can be threaded onto a linear polymeric chain. In a follow-up study, Ogoshi et al. reported an elegant polyrotaxane, capable of forming a topological gel by covalently linking alkene moieties on pillar[5]arene macrocycles [42]. Specifically, the pillar[5]arene macrocycles could function as CHLs. First, the authors utilised CHL1 wheels and polytetrahydrofuran as a polymer chain to prepare pillar[5]arene-based non-ionic polyrotaxanes. Then, by changing the terminal $-\mathrm{CH}_{3}$ groups on the TEO chains to crosslinkable $-\mathrm{CH}=\mathrm{CH}_{2}$ groups, topological gels were obtained after inter-molecular crosslinking using olefin metathesis. This case provides a new perspective to fabricate covalently cross-linked gels.

\section{Smart window materials}

Due to the thermo-responsiveness of TEO-modified pillar[6]arene (EGP6) and the redox-induced reversible color switching of ferrocene/ferrocenium, Wang et al. developed a novel hydrogel based smart window this year [43], Advanced functional materials play a pivotal role in the construction of smart windows, and can provide a more comfortable indoor environment for humans to enjoy a better lifestyle. Based on their previous research on hostguest interactions between pillar[6]arene and ferrocene [44-48], the authors first prepared a well-swollen and highly transparent hydrogel (Fc-gel-EGP6) which was obtained by immersing dry Fcgel in an aqueous solution of EGP6 (Fig. 8). Due to the formation of hydrophilic inclusion complexes between EGP6 and Fc-gel, the water absorption capacity of the generated Fc-gel-EGP6 hydrogel is dramatically improved, leading to drastically improved swelling behavior and an enhanced transparency. Furthermore, because of the thermo-responsiveness exhibited by EGP6 and the reversible transformation between the orange and green colors of the ferrocene/ferrocenium group under redox-conditions, they have orthogonally integrated these functional properties in the design of a warm/cool tone-switchable thermochromic material. The authors are confident that such materials are highly desirable for the fabrication of smart windows with dual functionality-both for regulating the input of solar energy and for improving the mood and emotions of the inhabitants of a room.

\section{Conclusions and outlook}

In conclusion, recent advances in the construction of dynamic functional materials based on water soluble pillar[n]arenes with TEO groups are summarized in this review. Pillar[n]arene with TEO substituents is an extremely versatile host in the pillar[n]arene family, and exhibits numerous advantageous properties, such as water solubility, thermoresponsivity and liquidity at room temperature. Thus the application of the many pillar[n]arenes with TEO groups described in this review includes thermoresponsive materials with LCST behavior, cyclic host liquids and smart windows. In the future, we believe that the properties of such pillar[n]arenes will be further explored and many more fascinating dynamic materials fabricated from TEO substituted pillar[n]arenes will be reported.

\section{Acknowledgments}

We gratefully thank the financial support from the National Natural Science Foundation of China (No. 21702020) and Maynooth University.

\section{References}

[1] M. Zhang, X. Yan, F. Huang, et al., Acc. Chem. Res. 47 (2014) 1995-2005.

[2] S. Dong, B. Zheng, F. Wang, et al., Acc. Chem. Res. 47 (2014) 1982-1994.

[3] T. Xiao, S.L. Li, Y. Zhang, et al., Chem. Sci. 3 (2012) 1417-1421.

[4] S.L. Li, T. Xiao, C. Lin, et al., Chem. Soc. Rev. 41 (2012) 5950-5968.

[5] L. Qin, P.F. Duan, M.H. Liu, Chin. Chem. Lett. 25 (2014) 487-490.

[6] X.J. Liao, G.S. Chen, Chin. Chem. Lett. 27 (2016) 583-587.

[7] Z. Qi, C.A. Schalley, Acc. Chem. Res. 47 (2014) 2222-2233.

[8] E.A. Appel, J. del Barrio, X.J. Loh, et al., Chem. Soc. Rev. 41 (2012) 6195-6214.

[9] Q. Wang, P. Zhang, Y. Li, et al., RSC Adv. 7 (2017) 29364-29367.

[10] Q. Wang, M. Cheng, L. Tian, et al., Polym. Chem. 8 (2017) 6058-6063.

[11] T. Ogoshi, T.A. Yamagishi, Y. Nakamoto, Chem. Rev. 116 (2016) 7937-8002.

[12] N.L. Strutt, H. Zhang, S.T. Schneebeli, et al., Acc. Chem. Res. 47 (2014) 2631-2642.

[13] M. Xue, Y. Yang, X. Chi, et al., Acc. Chem. Res. 45 (2012) 1294-1308.

[14] D. Cao, Y. Kou, J. Liang, et al., Angew. Chem. Int. Ed. 48 (2009) 9721-9723.

[15] T. Ogoshi, S. Kanai, S. Fujinami, et al., J. Am. Chem. Soc. 130 (2008) 5022-5023.

[16] J.C. Gui, Z.Q. Yan, Y. Peng, et al., Chin. Chem. Lett. 27 (2016) 1017-1021.

[17] X.S. Hu, H.M. Deng, J. Li, et al., Chin. Chem. Lett. 24 (2013) 707-709.

[18] B. Yuan, J.F. Xu, C.L. Sun, et al., ACS Appl. Mater. Interface 8 (2016) 3679-3685.

[19] T.T. Cao, X.Y. Yao, J. Zhang, et al., Chin. Chem. Lett. 26 (2015) 867-871.

[20] F. Sakai, Z.W. Ji, J.H. Liu, et al., Chin. Chem. Lett. 24 (2013) 568-572.

[21] Y. Zhou, H. Li, Y.W. Yang, Chin. Chem. Lett. 26 (2015) 825-828.

[22] L. Wang, M.O. Vysotsky, A. Bogdan, et al., Science 304 (2004) 1312-1314.

[23] D.S. Guo, Y. Liu, Acc. Chem. Res. 47 (2014) 1925-1934.

[24] H.L. Sun, Y. Chen, J. Zhao, et al., Angew. Chem. Int. Ed. 54 (2015) 9376-9380.

[25] S.A. Nepogodiev, J.F. Stoddart, Chem. Rev. 98 (1998) 1959-1976.

[26] D. Zhao, Y. Chen, Y. Liu, Chin. Chem. Lett. 26 (2015) 829-833.

[27] Y. Wang, G. Ping, C. Li, Chem. Commun. 52 (2016) 9858-9872.

[28] J. Roeser, F. Moingeon, B. Heinrich, et al., Macromolecules 44 (2011) 8925-8935.

[29] T. Ogoshi, R. Shiga, T.A. Yamagishi, J. Am. Chem. Soc. 134 (2012) 4577-4580. [30] G. Yu, M. Xue, Z. Zhang, et al. J. Am. Chem. Soc. 134 (2012) 13248-13251.

[31] G. Yu, X. Zhou, Z. Zhang, et al., J. Am. Chem. Soc. 134 (2012) 19489-19497.

[32] T. Ogoshi, T. Aoki, R. Shiga, et al., J. Am. Chem. Soc. 134 (2012) 20322-20325.

[33] T. Ogoshi, K. Kida, T.A. Yamagishi, J. Am. Chem. Soc. 134 (2012) 20146-20150.

[34] X. Chi, M. Xue, Chem. Commun. 50 (2014) 13754-13756.

[35] X. Chi, X. Ji, D. Xia, et al., J. Am. Chem. Soc. 137 (2015) 1440-1443.

[36] X. Chi, G. Yu, L. Shao, et al., J. Am. Chem. Soc. 138 (2016) 3168-3174.

[37] Y.L. Ma, H. Ke, A. Valkonen, et al., Angew. Chem. Int. Ed. 57 (2018) 709-713.

[38] G.B. Huang, W.E. Liu, A. Valkonen, et al., Chin. Chem. Lett. 29 (2018) 91-94.

[39] L.L. Wang, Z. Chen, W.E. Liu, et al., J. Am. Chem. Soc. 139 (2017) 8436-8439.

[40] F. Jia, Z. He, L.P. Yang, et al., Chem. Sci. 6 (2015) 6731-6738.

[41] L.P. Yang, H. Liu, S.B. Lu, et al., Org. Lett. 19 (2017) 1212-1215.

[42] T. Ogoshi, T. Aoki, S. Ueda, et al., Chem. Commun. 50 (2014) 6607-6609.

[43] S. Wang, Z. Xu, T. Wang, et al., Nat. Commun. 9 (2018) 1737-1745.

[44] S. Wang, C.H. Yao, M.F. Ni, et al., Polym. Chem. 8 (2017) 682-688.

[45] M. Ni, N. Zhang, W. Xia, et al., J. Am. Chem. Soc. 138 (2016) 6643-6649.

[46] W. Xia, M. Ni, C. Yao, et al., Macromolecules 48 (2015) 4403-4409.

[47] W. Xia, X.Y. Hu, Y. Chen, et al., Chem. Commun. 49 (2013) 5085-5087.

[48] Q. Duan, Y. Cao, Y. Li, et al., J. Am. Chem. Soc. 135 (2013) 10542-10549. 